\title{
Modeling of the Pulp Density with Artificial Neural Networks
}

\author{
M.Sc. Deynier Montero Góngora ${ }^{1^{*}}$, Eng. Yoandris Columbié Fornaris ${ }^{2}$, \\ Eng. Keiler Cobas Cardoza ${ }^{3}$ \\ ${ }^{1,2,3}$ Higher Mining Metallurgical Institute of Moa
}

*Corresponding Author: M.Sc. Deynier Montero Góngora, Higher Mining Metallurgical Institute of Moa

\begin{abstract}
This investigation approaches the artificial neural networks applied to the pulp sedimentation process in carbonate-ammonia leaching. To carry out this research, the main variables that characterize the process were identified. Besides, it was collected the data that comprise a whole month of facility's operation. Furthermore, it was developed a regression analysis backwards, step by step, which allowed to determine that the linear correlation coefficient did not reach values higher than 0,7. In addition, it was pinpointed a two layered feed -forward back propagation neural network to model the pulp density. Thins one reached the correlation coefficient values of 0,91 during its training and validation, as well as 0,67 in its generalization.
\end{abstract}

Keywords: artificial neuronal network, regression, feed-forward backpropagation, pulp sedimentation.

\section{INTRODUCTION}

In a global context, nowadays, modern control systems play a fundamental role when developing solutions to issues or problems presented in domestic and industrial applications. The main contributions of modern control systems at industrial level contribute to technological innovation, profitability and maintainability of the controlled processes.

Within the advanced control strategies under investigation to automate complex processes are: adaptive control, predictive control based on models, robust control, and intelligent control, among others. Intelligent control relies on several techniques such as: fuzzy logic, evolutionary algorithms, and artificial neural networks.

Artificial neural networks can be used effectively and accurately for modeling systems with complex dynamics, especially for nonlinear processes that vary over time. The growing interest in neural networks is due to its great versatility and the continuous advance in network training algorithms and hardware [1], [2], [3], [4].

The nickel producing companies have continuous processes of great complexity that require automation to achieve a greater efficiency in their productions. In the carbonate-ammonia leaching scheme of reduced ore, certain specifications must be met: the discharge in pulp settlers must be regulated in such a way that, even when removing sludge with the lowest possible humidity, substantially, the same amount of solids with which the settler is fed every day should be removed in a thickened mud form. If the feeding to the settler suffers a significant variation, the discharge must be regulated to remove an amount of mud approximately equal to the average amount of solids entering the thickener as frequent pumping changes are undesirable. Therefore, it is important to maintain a control of the pulp density at the exit of the settlers [5].

There is insufficient knowledge on the part of the operators on how to control the main variables registered in the settler. There have occurred faults that have prevented the continuous operation of the SD-109A settler tank due to the high ore agglomeration in all structures of the truss and arms. Hence, there has been uneven torque in the arms and the accumulation of solids in the feeder's upper and lower chambers. In addition, a high solid obstruction was detected in the entrance line to the upper chamber of the feeder.

The inefficiencies in the control of the pulp density in the bottom of the SD-109A settler tank are taken as a research problem and as an objective to obtain an artificial neural model for the pulp 
density in the bottom of the Settler tank on the basis of the main input variables, using Mat lab as a calculation tool.

\section{MATERIALS AND METHODS}

\subsection{Description of the Pulp Sedimentation Process}

The solids settle to form a pulp with a density of $1700-1750 \mathrm{~g} / \mathrm{L}$, which is moved by the settler tank arms (Figure 1) towards the center of it. There, it is sucked and pumped towards two settler tanks, acting as a second stage of sedimentation (passive stage of leaching)), in which the pulp does not receive magnetic flocculation or aeration, only phase separation. The overflow of the second stage settler tanks is sent completely through centrifugal pumps to the collector tank where it is mixed with the part of first stage liquor, which are then cooled and destined to be used as recirculating liquor in the contact channels.

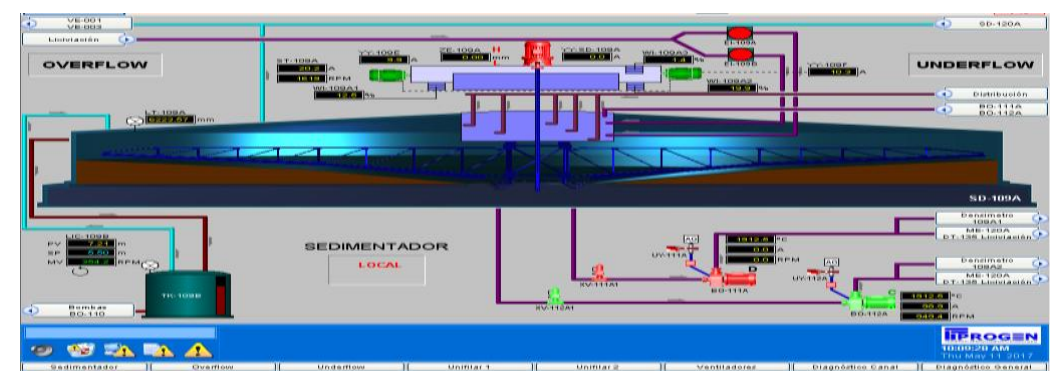

Figure1. Schematic diagram of the settler tank

The bottom pulp of the settler tanks of the third stage is sent to the washing area. This area is composed of two series, (A and B), with five settler tanks each which are called washing stage. Here the pulp receives magnetic flocculation through electromagnets located in the middle of each of these stages to increase their sedimentation rates. This is used in all washing stages in order to achieve a better phase separation (liquid-solid) in each settler tank [5].

The pulp settler tank is a complex physical-mathematical modeling object with a large number of input and output parameters which are in a complex interdependence (Figure 2).

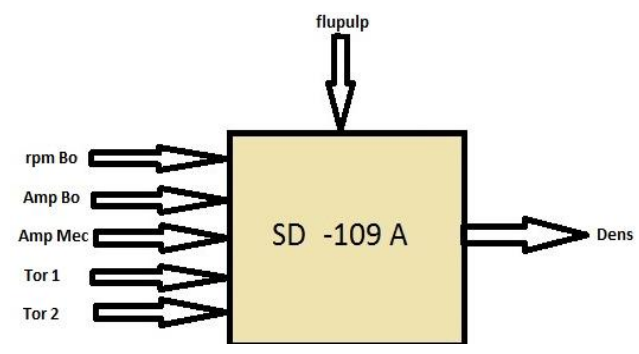

Figure2. Scheme of the pulp settler tank.

The parameters in the process are:

- $\mathrm{rpmBo} \Rightarrow$ Feed motor speed [rpm]

- $\mathrm{AmpBo} \Rightarrow$ Pump power Bo-112A $[\mathrm{A}]$

- AmpMec $\Rightarrow$ Power in the motors of the main mechanism $[\mathrm{A}]$

- Tor $1 \Rightarrow$ Torque in motor 1 (Wt-109 A1) of scraper arms [lblft]

- Tor $2 \Rightarrow$ Torque in motor 2 (Wt-109 A2) of scraper arms [lblft]

input

\section{The output parameter is:}

- Dens $\Rightarrow$ Pulp Density [g/L]

In addition to the input and output parameters, it is important to highlight a specific disturbance of this process that influences it, which is:

Flujopulp $\Rightarrow$ Flow of pulp fed to the Settler tank $[\mathrm{Kg} / \mathrm{h}]$.

International Journal of Innovative Research in Electronics and Communications (IJIREC) 
Due to the automation existing in the process, the values of the process parameters are sensed by the instrument corresponding to each of them and the signal is sent to the computer located in the process control office. The data obtained along 1 month of operation, were recorded every $48 \mathrm{~s}$ and processed with the Stat graphics Plus V 5.1 software.

\subsection{Artificial Neural Networks}

The determination of the type of artificial neural network, the number of layers and the number of neurons in each layer that best characterize the process of pulp sedimentation was carried out through a trial and error process that plays with the number of neurons and the maximum permissible error.

Through Matlab's Toolbox (nnstart), the performance of artificial neural models was evaluated by using the mean square error and the correlation coefficient between the real values and those obtained by the network [6]. The objective was to provide the network with an adequate number of neurons in the hidden layer to learn about the characteristics of the possible relationships between the sample data. Through the trial and error process, it was identified the feed-forward back propagation structure that provided better results.

The proposed network consists of two layers: a hidden layer and an output layer. The output layer will only have one unit, which will indicate the value of the output pulp density associated with each input vector presented to the network. The hidden layer will have a variable number of neurons. Schematically, the proposed model of artificial neuron network to estimate the density of pulp in the bottom of the SD-109 A settling tank has the shape shown in Figure 3.

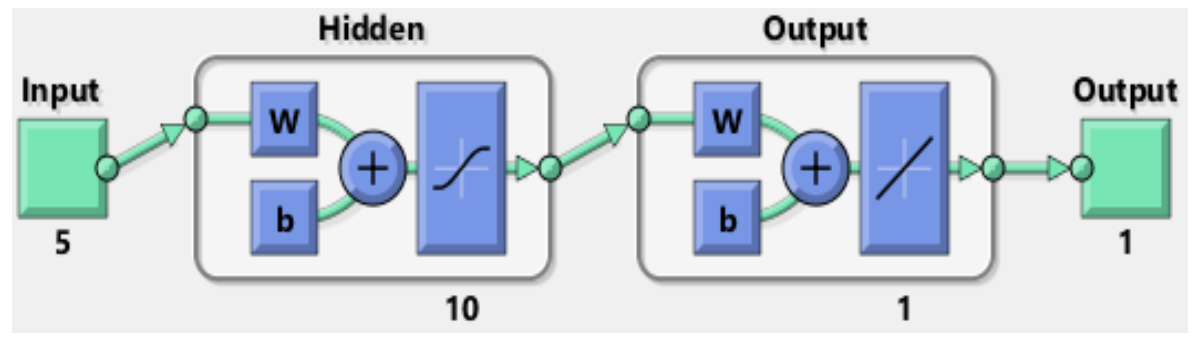

Figure3. Schematic representation of the artificial neuron network used.

Table 1 shows the input variables to the artificial neural network, which will be used, by following that order, in the training, validation and generalization of it.

Table1. Summary of data for the artificial neuron network.

\begin{tabular}{|l|l|l|l|l|l|l|}
\hline No. & rpmBo & AmpBo & AmpMec & Tor 1 & Tor 2 & Dens \\
\hline $\mathbf{1}$ & $\mathrm{Rpm}_{1}$ & $\mathrm{AmpBo}_{1}$ & $\mathrm{AmpMec}_{1}$ & Tor $1_{1}$ & Tor $2_{1}$ & Dens $_{1}$ \\
\hline $\mathbf{2}$ & $\mathrm{Rpm}_{2}$ & $\mathrm{AmpBo}_{2}$ & $\mathrm{AmpMec}_{2}$ & Tor $1_{2}$ & Tor $2_{2}$ & Dens $_{2}$ \\
\hline $\mathbf{3}$ & $\mathrm{Rpm}_{3}$ & $\mathrm{AmpBo}_{3}$ & $\mathrm{AmpMec}_{3}$ & Tor $1_{3}$ & Tor $2_{3}$ & Dens $_{3}$ \\
\hline $\mathbf{n}$ & $\mathrm{Rpm}_{\mathrm{n}}$ & $\mathrm{AmpBo}_{\mathrm{n}}$ & $\mathrm{AmpMec}_{\mathrm{n}}$ & Tor $1_{\mathrm{n}}$ & Tor $2_{\mathrm{n}}$ & Dens $_{\mathrm{n}}$ \\
\hline
\end{tabular}

The dependent variable (Dens: Pulp Density) is subjected to the five input variables

Dens $=f(A m p B o$, Ampmec, rpmBo, Tor 1, Tor 2$)$

\section{RESULTS AND DISCUSSION}

Figure 4 shows the behavior of the pulp density at the bottom of SD-109 A Settler tank between its minimum and maximum values 1673 and $1693 \mathrm{~g} / \mathrm{L}$ respectively, for the month of operation.

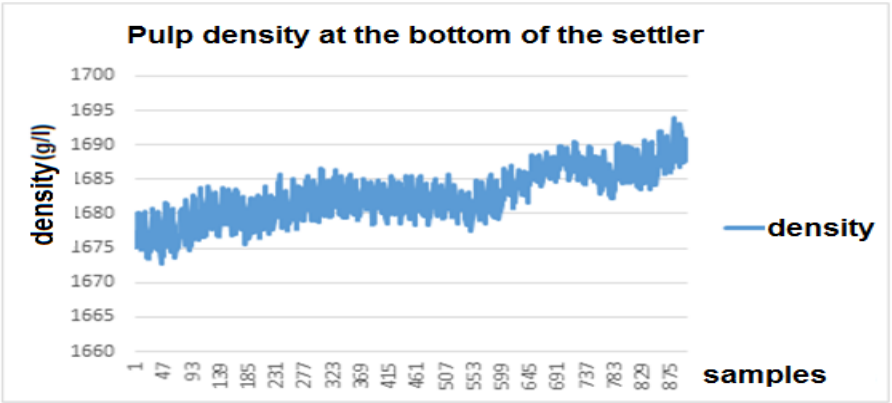

Figure4. Control chart for the dependent variable. 
Once the database was analyzed, the sample functions that evaluate the measures of central tendency and dispersion of the sample were determined through a descriptive statistical analysis (See Table 2).

For this case, it is observed that the Curtosis values and the Asymmetry coefficient, allow to establish that the dependent variable (pulp density) behaves as a normal distribution.

Table2. Summary of the sample's descriptive statistical analysis for one month

\begin{tabular}{|l|c|c|c|c|c|c|}
\hline \multicolumn{1}{|c|}{ Estadísticas } & $\begin{array}{c}\text { Dens } \\
(\mathrm{g} / \mathrm{L})\end{array}$ & $\begin{array}{c}\text { Amp Bo } \\
(\mathrm{A})\end{array}$ & $\begin{array}{c}\text { Rpm Bo } \\
(\mathrm{rpm})\end{array}$ & $\begin{array}{c}\text { Tor1 } \\
(\mathrm{lb} \backslash \mathrm{ft})\end{array}$ & $\begin{array}{c}\text { Tor2 } \\
(\mathrm{lb} \backslash \mathrm{ft})\end{array}$ & $\begin{array}{c}\text { Amp Mec } \\
(\mathrm{A})\end{array}$ \\
\hline Average & 1682,7 & 104,4 & 938,7 & 11,9 & 12,8 & 20,03 \\
\hline Standard deviation & 3,9 & 1,8 & 2,2 & 2,3 & 2,2 & 0,04 \\
\hline Variance & 15,2 & 3,5 & 4,97 & 5,5 & 4,8 & 0,001 \\
\hline Curtosis & $-0,4$ & $-1,1$ & 14,5 & $-1,3$ & $-1,2$ & $-0,5$ \\
\hline Asymmetry coefficient & 0,15 & $-0,14$ & $-1,7$ & $-0,03$ & 0,04 & 0,19 \\
\hline Minimum & 1672,7 & 97,6 & 914,4 & 7,7 & 9,3 & 19,9 \\
\hline Maximum & 1693,9 & 107,6 & 942,5 & 16 & 17,3 & 20,1 \\
\hline Confidence interval (95 \%) & 0,2 & 0,12 & 0,14 & 0,15 & 0,14 & 0,002 \\
\hline
\end{tabular}

The mathematical model that best represents the relationship between the variables analyzed is shown.

Dens $=1233,7+0,58 * A m p B o+17,5 *$ AmpMec $-0,96 * r p m B o+1,9 *$ Tor $1+1,8 *$ Tor 2

Table 3 shows the regression analysis for the output pulp density, where a 0,7 correlation coefficient is observed.

Table3. Regression analysis summary

\begin{tabular}{|c|c|}
\hline Correlation coefficient R & 0,7 \\
\hline Adjusted R & 0,5978 \\
\hline Average absolute error & 1,9872 \\
\hline Standard error & 2,4748 \\
\hline Durbin-Watson Statistic & 0,5507 \\
\hline Auto correlation of waste in delay 1 & 0,7223 \\
\hline
\end{tabular}

Figure 5 shows the behavior of the correlation coefficients for the training, validation, testing and adjustment of the artificial neuron network (it is assumed as an artificial neuronal model for the pulp density in the settler tank bottom "nnDens" and the real density "Dens").

Figure 6 shows the generalization of the network with 902 data not presented during training, where a 0,66 correlation coefficient is observed.
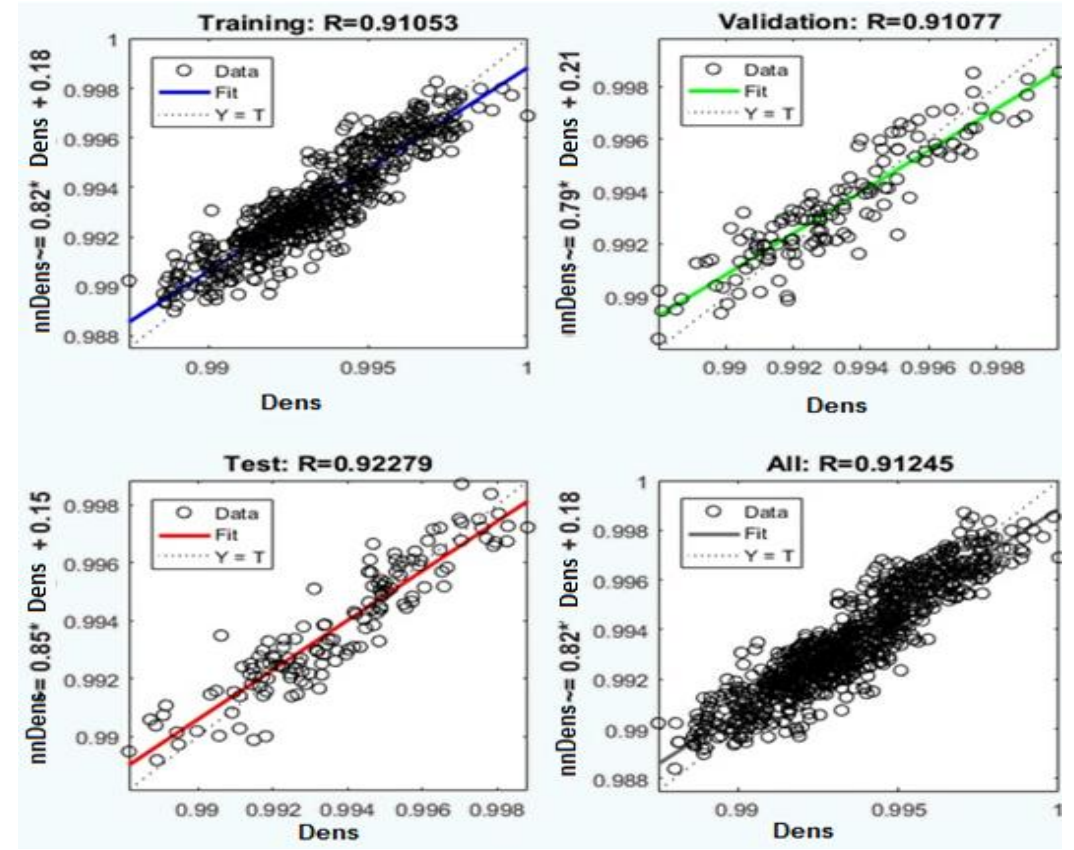

Figure5. Correlation coefficients of the neural network. 


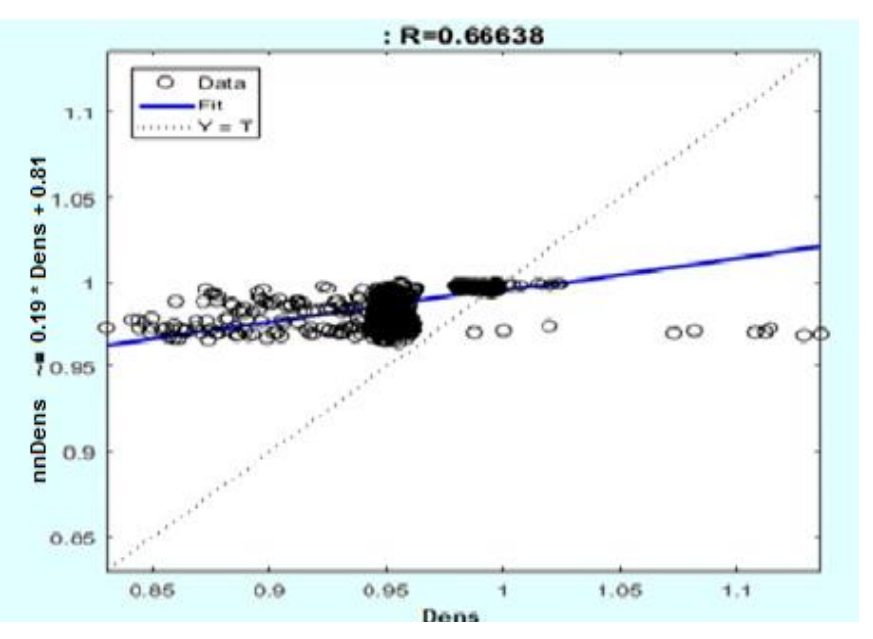

Figure6. Network Generalization

\section{CONClusion}

The capacity of the feed-forward back propagation network for the simulation of pulp sedimentation processes in the industry was demonstrated.

The structure that best characterizes the behavior of pulp density in the settler tank is characterized by two layers with 10 neurons in the hidden layer and one in the output layer, with the LevenbergMarquart learning method (trainlm), and the log-sigmoidal (logsig) and linear (pureline) transfer functions.

\section{ACKNOWLEDGMENTS}

To the Lice. Yanisel Batista Nunez for her valuable contribution in this paper's translation.

\section{REFERENCES}

[1] Ljung, L, Sjöberg, J (1992). IEEE Workshop on Neural Networks for Signal. Processing. IEEE Service Center, 445 Hoes Lane, Piscataway, NJ 08854-4150.

[2] Isermann, R., Ayoubi, M., Konrad, H., \& Reiss, T (1993). tools, Model based detection of tool wear and breakage for machine.

[3] Valverde, R. y. (2007). Identificación de sistemas dinámicos utilizando redes neuronales RBF. RIAI, 4(2), $32-42$.

[4] Santos, M (2011). Un Enfoque Aplicado del Control Inteligente. RIAI, 8, 283-296.

[5] Manual de Operaciones (2015). UBP “Lixiviación”. Cmdte. Ernesto Che Guevara, Moa.

[6] Spencer, R (2009). Identification of the ore cooling procedure in the CARON process, with Artificial Neural Networks. Master dissertation, ISMMM.

\section{AUTHORS' BIOGRAPHY}

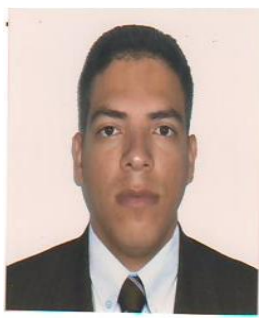

Deynier Montero Góngora, Master's Degree in Electro-Mechanics; Higher Mining Metallurgical Institute of Moa, Holguin, Cuba

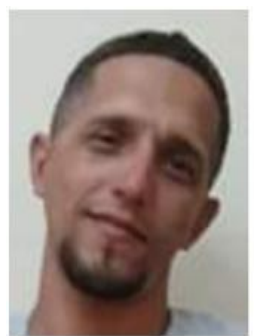

Yoandris Columbié Fornaris, Electrical Engineer; Higher Mining Metallurgical Institute of Moa, Holguin, Cuba 

Keiler Cobas Cardoza, Automation Engineer; Higher Mining Metallurgical
Institute of Moa, Holguin Cuba

Citation: Deynier Montero Góngora, et.al. (2018) "Modeling of the Pulp Density with Artificial Neural Networks", International Journal of Innovative Research in Electronics and Communications (IJIREC), 5(1), pp 18-23. DOI: http://dx.doi. org/10.20431/2349-4050.0501004

Copyright: (C) 2018 Deynier Montero Góngora, This is an open-access article distributed under the terms of the Creative Commons Attribution License, which permits unrestricted use, distribution, and reproduction in any medium, provided the original author and source are credited. 